\title{
RX J004717.4-251811: The first eclipsing X-ray binary outside the Local Group ${ }^{\star}$
}

\author{
W. Pietsch, F. Haberl, and A. Vogler \\ Max-Planck-Institut für extraterrestrische Physik, 85741 Garching, Germany \\ Received 19 December 2002 / Accepted 25 February 2003

\begin{abstract}
The X-ray source RX J004717.4-251811 in the nearby starburst galaxy NGC 253 was found to undergo changes from a low to a high state twice, during an XMM-Newton EPIC observation in December 2000 and also during a Chandra observation one year earlier. These transitions are interpreted as egresses from eclipses of a compact object in a high mass $\mathrm{X}$-ray binary system (HMXB). The phase of eclipse egress during the Chandra observation is given by barycenter corrected two egresses and number $\mathrm{n}$ of periods in-between. Allowed periods may be further constrained by additional XMM-Newton, Chandra, ROSAT, and Einstein observations resulting in only seven acceptable periods with $1.47024 \mathrm{~d}$ and $3.20793 \mathrm{~d}$ most promising. No significant regular pulsations of the source in the range 0.3-1000 s were found. Fluctuations on time scales of $1000 \mathrm{~s}$ were observed together with extended intervals of low intensity. The energy spectrum during the bright state can best be described by an absorbed flat power law $\left(N_{\mathrm{H}}=1.9 \times 10^{21} \mathrm{~cm}^{-2}, \Gamma=1.7\right)$. In the bright state the source luminosity is $4 \times 10^{38}(0.5-5 \mathrm{keV})$, just compatible with the Eddington luminosity of a $1.4 M_{\odot}$ neutron star. A possible optical identification
\end{abstract} \\ MJD $51539.276 \pm 0.006$ and the binary period determined to $p=(352.870 \pm 0.012) \mathrm{d} / n$ by the time difference between the \\ is suggested. RX J004717.4-251811 parameters are compared to other eclipsing X-ray binaries (XRBs).
}

Key words. galaxies: individual: NGC 253 - X-rays: stars - stars: binaries: eclipsing

\section{Introduction}

Luminous X-ray binaries (XRBs) in the Milky Way were among the first X-ray sources detected and optically identified. They are classified as low mass X-ray binary (LMXBs) and high mass X-ray binary (HMXBs) systems according to the mass of the donor star. The accreting collapsed object can be a neutron star or a black hole in either class.

XRBs may show orbital variability (eclipses, absorption dips, regular outbursts), pulsations due to rotation of the compact object (neutron star), long term variability (which may be explained due to precession of an accretion disk or free precession of a neutron star) and flares. Examples for eclipsing XRBs are Her X-1, LMC X-4, SMC X-1 with pulsation periods of $1.24 \mathrm{~s}, 0.71 \mathrm{~s}, 13.5 \mathrm{~s}$, orbital periods of $1.7 \mathrm{~d}, 1.4 \mathrm{~d}, 3.9 \mathrm{~d}$, full X-ray eclipse durations of at least $0.137,0.144,0.147$ in phase, long term periods of $35 \mathrm{~d}, 30 \mathrm{~d}, 45 \mathrm{~d}-60 \mathrm{~d}$, and showing no flares, flares, no flares, respectively (Liu et al. 2000, and references therein). Eclipsing XRBs up to now were only detected within the Local Group and the total number remains small. The first examples of such systems (Cen X-3 and Her X-1: Schreier et al. 1972b; Tananbaum et al. 1972,

Send offprint requests to: W. Pietsch, e-mail: wnp@mpe.mpg. de

* This work is based on observations obtained with XMM-Newton, an ESA Science Mission with instruments and contributions directly funded by ESA Member States and the USA (NASA). respectively), were detected in the Milky Way with the UHURU satellite, then in the SMC (SMC X-1: Schreier et al. 1972a) and LMC (LMC X-4: Li et al. 1978; White 1978), and finally one in M 33 (Peres et al. 1989). Combining X-ray pulse arrival time information and orbital parameters of pulsating eclipsing XRBs with radial-velocities of the optical companion were essential to determine neutron star masses (see e.g. van Paradijs \& McClintock 1995).

In this paper we report on the first detection of an eclipsing XRB outside the Local Group in NGC 253. This prototypical nearby edge-on starburst galaxy was observed by many X-ray satellites. X-ray point sources could be resolved with the high spatial resolution instruments aboard Einstein, ROSAT, Chandra, and XMM-Newton. Fabbiano \& Trinchieri (1984, hereafter FT84) reported complex X-ray emission from eight point sources as well as diffuse emission from nuclear area, plume and disk using the Einstein HRI detector. Vogler \& Pietsch (1999, hereafter VP99) analyzed X-ray point sources in the bulge, disk and halo of NGC 253 in ROSAT PSPC and HRI observations. The number of point-like $\mathrm{X}$-ray sources increased to 30 sources, 13 of which are time variable. RX J004717.4-251811 (E2 of FT84, X17 of VP99) is one of the four Einstein sources detected again with ROSAT. During most of the ROSAT observations a second source of similar brightness (X18) was visible just $15^{\prime \prime}$ to the south. RX J004717.4-251811 shows strong time variability within the 
ROSAT observations and also compared to the Einstein HRI detection. This time variability and the ROSAT PSPC hardness ratio classification made RX J004717.4-251811 a good XRB candidate.

NGC 253 was observed by XMM-Newton as a performance verification target in June 2000. First results on bright point sources were discussed in Pietsch et al. (2001, hereafter P01). Of special interest was the spectrum and time variability of the ROSAT source X33 $30^{\prime \prime}$ south of the nucleus, most likely a black hole XRB. The pair of ROSAT sources (X17/X18) is clearly visible in Fig. 1 of P01, however deferred for later analysis, as these sources at the time could not be distinguished by the SAS source detection algorithms.

NGC 253 was again observed by us with XMM-Newton in December 2000 within the XMM-Newton survey scientist guaranteed time program. We noticed that during this observation the count rate of RX J004717.4-251811 abruptly changed from very low to high. This behavior most likely represents an egress from an eclipse by the companion star in this XRB. Triggered by this finding we analyzed archival Chandra, XMM-Newton, ROSAT, and Einstein observations. This led to the detection of a similar behavior during Chandra observation 790. Data from all the satellites were then used to further constrain the binary orbit parameters and long term time variability of the source. In the following we report on these findings.

\section{Observations}

For the detailed analysis of RX J004717.4-251811 we used data from XMM-Newton, Chandra, ROSAT HRI, and Einstein. Table 1 summarizes the observatory (Col. 1), observation identification (2), observation dates (3) and life time (4), extraction radius $R_{\mathrm{e}}$ used for XMM-Newton and Chandra count rates and light curves and spectra (5), RX J004717.4-251811 count rates (6), hardness ratios for XMM-Newton EPIC PN and Chandra ACIS S (7), and luminosities in the $0.5-2.4 \mathrm{keV}$ band (8), an energy band covered by all observatories. Special events are marked under comments (9). As hardness ratio (HR) we use the ratio of the counts in the $1.5-5.0 \mathrm{keV}$ band to the counts in the $0.5-1.5 \mathrm{keV}$ band.

During the XMM-Newton observations (Jansen et al. 2001) the EPIC PN and MOS instruments (Strüder et al. 2001; Turner et al. 2001) were operated in the full frame mode resulting in a time resolution of $73.4 \mathrm{~ms}$ and $2.6 \mathrm{~s}$, respectively. The medium filter was in front of the EPIC MOS1 camera in all observations, for MOS2, the thin filter was used during the observations in June 2000 and the medium filter in December. The PN camera operated with the medium filter during observation 0125960101 and with the thin filter for the other observations. These filter changes do not influence the RX J004717.4251811 observations significantly as source counts are mainly detected above $0.7 \mathrm{keV}$ (see below). In this energy band the transmission of the thin and medium filter do not differ significantly. We used all EPIC instruments for the imaging and position determination of RX J004717.4-251811. For the timing and spectral investigations we concentrated on the EPIC PN camera which gives about twice the number of photons and checked the results for consistency and at the beginning of the observing window using the MOS cameras. While the XMMNewton point spread function (PSF) normally requires extraction radii $>30^{\prime \prime}$ to encircle $>80 \%$ of the source photons, we had to restrict ourselves to $10^{\prime \prime}$ to avoid contamination from source VP99 X18 (see Fig. 3).

Three Chandra ACIS S observations of NGC 253 (see Table 1) were obtained from the Chandra Data Archive (http://asc.harvard.edu/cgi-gen/cda). The instrument was operated in the full frame mode $(3.2 \mathrm{~s}$ time resolution). RX J004717.4-251811 is positioned in the backilluminated CCD chip S3 during observation 969 and 383, in the front-illuminated chip S2 during 790. Due to the larger offaxis position of RX J004717.4-251811 in the latter observation, the Chandra PSF urged us to use a larger extraction radius.

The deep space orbits of the satellites XMM-Newton and Chandra led to long observation times of RX J004717.4251811 without interruption. The low earth orbits of the Einstein and ROSAT satellite on the other hand, led to observations split in many short intervals of typically less than $1500 \mathrm{~s}$.

NGC 253 was observed with the ROSAT PSPC and HRI in several observing runs from Dec. 1991 to Dec. 1998. We restricted our analysis on $75.5 \mathrm{ks}$ HRI observations as only this ROSAT detector fully resolves RX J004717.4-251811 from VP99 X18. The HRI observation 601113h (July 1998, 2 ks) could not be used for the investigations due to attitude problems.

RX J004717.4-251811 cannot be resolved from nearby sources with the Einstein IPC. However, the source was clearly detected with the HRI (FT84) during a bright state. The nearby source VP99 X18 was not detected. For additional timing analysis we extracted the NGC 253 HRI data from the Einstein Data Archive at HEASARC.

The data analysis was performed using tools in the SAS v5.3.3, CIAO v2.2, EXSAS/MIDAS 1.2/1.4, and FTOOLS v5.1 software packages, the imaging application DS9 v2.1, the timing analysis package XRONOS v5.19 and spectral analysis software XSPEC v11.2.

For the time variability investigations all RX J004717.4251811 event times were corrected to solar system barycenter arrival times.

\subsection{Time variability}

The EPIC PN as well as the MOS light curves for XMM-Newton observation 0110900101 show egresses from low to high state at solar system barycenter corrected MJD 51892.146 \pm 0.006 (for EPIC PN see Fig. 1) with residual emission during low state which partly may be contaminated from emission collected from the PSF wing of VP99 X18. During the high state the average HR was 0.86 and constant within the errors (average HR 0.86 , see Table 1), the HR during the low state $(0.5)$ indicates a softer spectrum and/or less absorption. During each of the XMM-Newton observations 0125960101 and 0125960201 the flux was constant within the errors (see Table 1) and the source was a factor of 1.9 brighter than in the 0110900101 low state, a factor of more than five 
Table 1. XMM-Newton, Chandra, ROSAT, and Einstein observatory observations of RX J004717.4-251811. XMM-Newton count rates, hardness ratios (HR) and luminosities are determined for the EPIC PN camera.

\begin{tabular}{|c|c|c|c|c|c|c|c|c|}
\hline Observatory & Obs. id. & Obs. dates & $\begin{array}{l}\text { L.t. } \\
\text { (ks) } \\
(4)\end{array}$ & $\begin{array}{l}R_{\mathrm{e}} \\
\left({ }^{\prime \prime}\right) \\
(5)\end{array}$ & $\begin{array}{l}\text { Count rate } \\
\left(\mathrm{ct} \mathrm{ks}^{-1}\right) \\
(6)\end{array}$ & (7) & $\begin{array}{c}L_{\mathrm{X}}^{* *} \\
\left(10^{37} \mathrm{erg} \mathrm{s}^{-1}\right) \\
(8)\end{array}$ & Comment \\
\hline XMM-Newton & 0125960101 & 2000-06-03 & 39.0 & 10.0 & $7.0 \pm 0.4$ & $0.51 \pm 0.05^{\dagger}$ & $2.6 \pm 0.2$ & \\
\hline XMM-Newton & 0125960201 & 2000-06-04 & 14.0 & 10.0 & $7.2 \pm 0.7$ & $0.38 \pm 0.05^{\dagger}$ & $2.7 \pm 0.3$ & \\
\hline XMM-Newton & 0110900101 & $2000-12-13 / 14$ & 30.7 & 10.0 & $\begin{array}{r}4.3 \pm 0.7 \\
41.8 \pm 1.7\end{array}$ & $\begin{array}{l}0.50 \pm 0.15 \\
0.86 \pm 0.07\end{array}$ & $\begin{array}{r}1.4 \pm 0.2 \\
14.0 \pm 0.6\end{array}$ & $\begin{array}{l}\text { low state } \\
\text { high state }\end{array}$ \\
\hline Chandra & 969 & $1999-12-16$ & 14.2 & 3.5 & $54.8 \pm 2.0$ & $0.74 \pm 0.06$ & $20.1 \pm 0.7$ & \\
\hline Chandra & 790 & $1999-12-27$ & 44.1 & 5.0 & $\begin{array}{r}1.4 \pm 0.4 \\
28.7 \pm 0.9\end{array}$ & $\begin{array}{l}0.27 \pm 0.18 \\
0.91 \pm 0.06\end{array}$ & $\begin{array}{r}0.7 \pm 0.2 \\
14.6 \pm 0.5\end{array}$ & $\begin{array}{l}\text { low state } \\
\text { high state }\end{array}$ \\
\hline Chandra & 383 & 2001-08-16 & 2.2 & 3.5 & $4.6 \pm 1.5$ & $0.43 \pm 0.30$ & $1.6 \pm 0.5$ & \\
\hline ROSAT & $600088 \mathrm{~h}-0$ & $1991-12-08 / 10$ & 3.1 & 6.0 & $3.4 \pm 1.1$ & & $22 \pm 7$ & \\
\hline ROSAT & $600088 \mathrm{~h}-1$ & 1992-06-05/07 & 24.9 & 6.0 & $0.9 \pm 0.2$ & & $6 \pm 1$ & \\
\hline ROSAT & $600714 \mathrm{~h}$ & $1995-01-03 / 07$ & 10.9 & 6.0 & $4.0 \pm 0.6$ & & $26 \pm 4$ & \\
\hline ROSAT & $600714 \mathrm{~h}-1$ & $1995-06-13 / 17$ & 14.0 & 6.0 & $0.3 \pm 0.2$ & & $2 \pm 1$ & \\
\hline & & $1995-07-05 / 07$ & 5.9 & 6.0 & $0.3 \pm 0.2$ & & $2 \pm 1$ & \\
\hline ROSAT & $601111 \mathrm{~h}$ & $1997-12-20 / 27$ & 16.8 & 6.0 & $1.1 \pm 0.3$ & & $7 \pm 2$ & \\
\hline Einstein & $583 / 2083$ & $1979-07-05 / 08$ & 27.4 & 11.5 & $1.5 \pm 0.3^{*}$ & & $18 \pm 4$ & \\
\hline
\end{tabular}

Notes and references:

* According to Fabbiano \& Trinchieri (1984).

$\dagger$ The slightly higher HR during observation 0125960101 compared to 0125960201 reflects the difference between medium and thin filter and most likely not a change of the spectrum of RX J004717.4-251811.

** $0.5-2.4 \mathrm{keV}$ absorption corrected luminosity assuming an absorbed power law spectrum $\left(N_{\mathrm{H}}=1.9 \times 10^{21} \mathrm{~cm}^{-2}\right.$, photon index $\left.\Gamma=1.7\right)$ and a distance of NGC 253 of $2.58 \mathrm{Mpc}$ (Puche et al. 1991) which we use throughout the paper.

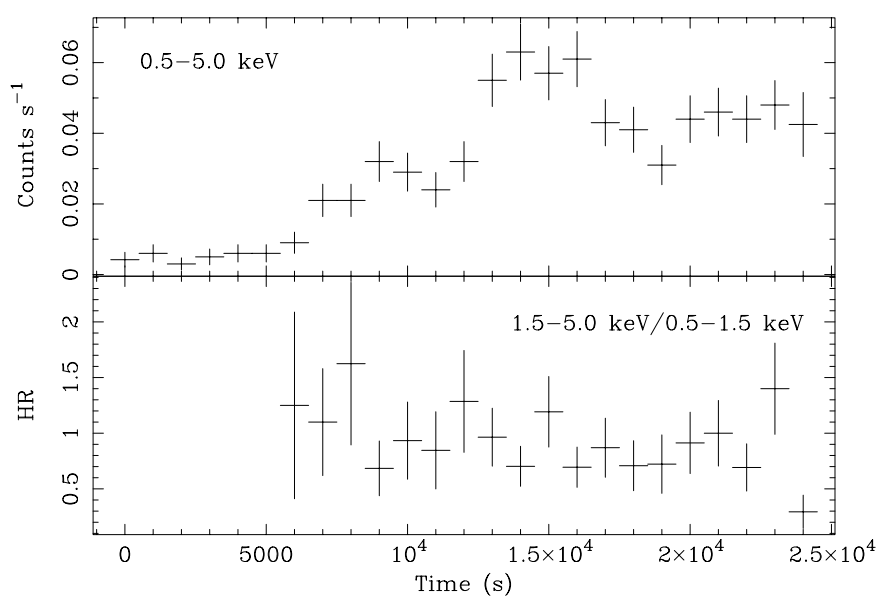

Fig. 1. XMM-Newton EPIC PN light curve and hardness ratio of observation 0110900101 integrated over $1000 \mathrm{~s}$. Time zero corresponds to MJD 51892.071251 (solar system barycenter corrected).

below that in the 0110900101 high state. The HRs during these observations on the other hand, appear to be quite similar to the 0110900101 low state.

The light curve for the Chandra observation 790 shows an egress from low to high state at solar system barycenter corrected MJD 51539.276 \pm 0.006 (Fig. 2). During the high state the HR defined in the same energy bands as for XMMNewton EPIC, was 0.91 and again constant within the errors. The residual emission during the low state clearly originates from RX J004717.4-251811 as is demonstrated in the right

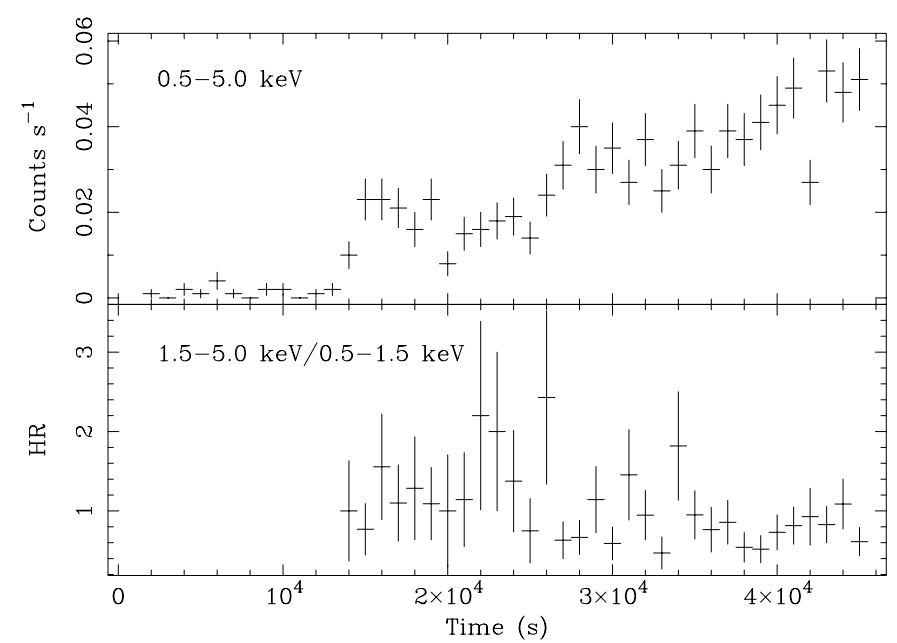

Fig. 2. Chandra ACIS S light curve and hardness ratio of observation 790 integrated over $1000 \mathrm{~s}$. Time zero corresponds to MJD 51539.114225 (solar system barycenter corrected).

part of Fig. 3 and corresponds to a count rate of $1.4 \mathrm{ct} \mathrm{ks}{ }^{-1}$. The HR again is significantly smaller than during the high state. The low state flux was about a factor of 2 below that in the XMM-Newton low state. During Chandra observation 969, RX J004717.4-251811 flux and HR were constant within the errors and the source about a factor of 1.4 brighter with similar average HR than that measured in the high state of 790 . During Chandra observation 383 the RX J004717.4-251811 flux was a factor of 12 below that during 969, but a factor 

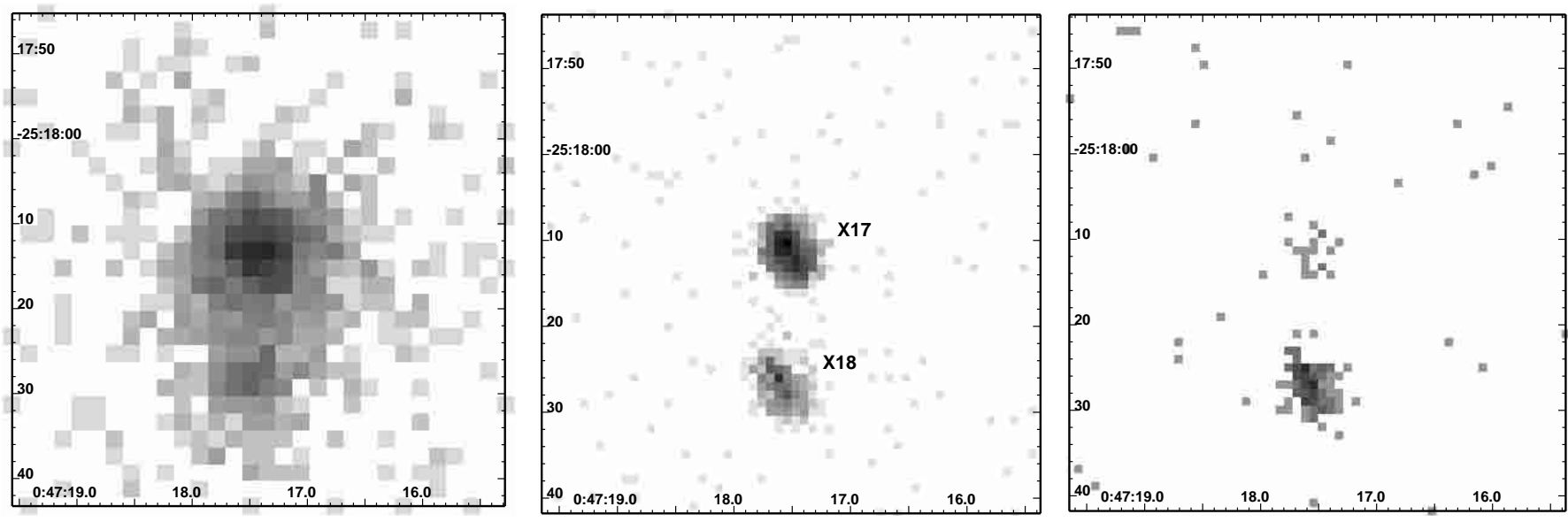

Fig. 3. Logarithmically-scaled, grey-scale images of the RX J004717.4-251811 region of the XMM-Newton EPIC high state in observation 0110900101 and Chandra ACIS S high and low state in observation 790 (from left to right). The images (RA, Dec J2000.0) were binned with a pixel size of $2^{\prime \prime}, 1^{\prime \prime}, 1^{\prime \prime}$ in the $0.5-5.0 \mathrm{keV}$ band for times after $8500 \mathrm{~s}$ in Fig. 1, after $14500 \mathrm{~s}$ and before $13500 \mathrm{~s}$ in Fig. 2, and have maxima of 66, 92, and 9 counts per pixel, respectively. The sources X17 (RX J004717.4-251811) and X18 are labeled in the center image.

of 2.3 higher than in the 790 low state, and the source showed a small HR (see Table 1). However, one has to keep in mind that the Chandra ACIS S count rates and HRs of RX J004717.4251811 cannot directly be compared to the others as they originate from a CCD with a different energy response function. The same is even more true if one wants to compare Chandra and XMM-Newton count rates and HRs.

Figure 3 shows images ( 1 ' to a side) of the RX J004717.4251811 area in the $0.5-5.0 \mathrm{keV}$ band. For XMM-Newton all EPIC cameras are combined for the high state of observation 0110900101. RX J004717.4-251811 is the source to the north, VP99 X18 the one to the south. The Chandra ACIS S images of observation 790 are divided in a low and high state image. RX J004717.4-251811 is clearly detected also during the low state.

We searched for pulsations within the bright parts of the XMM-Newton and Chandra observations in the frequency range $10^{-3}-3 \mathrm{~Hz}$. The strongest signal was found at $29.5 \mathrm{~s}$ in the XMM-Newton observation 0110900101 which, following the Rayleigh $Z_{n}^{2}$ method (Buccheri et al. 1983) as described in Haberl \& Zavlin (2002) yields only a $71 \%$ confidence level $(\sim 1 \sigma)$.

During the ROSAT HRI observations covering 6 years, 110 photons were detected within 6" from RX J004717.4251811 in an effective observing time of $75.5 \mathrm{ks}$. Due to the low ROSAT HRI background less than 10 of these counts may be due to field background. The source photons were not distributed evenly over the observing time but clearly showed strong intensity changes and even short term flares as is demonstrated by a Kolmogorov-Smirnov test against constancy (see Fig. 4).

\subsection{Improved position}

VP99 give the position of RX J004717.4-251811 with an error of 2 . 7 which is mostly determined by the included systematic error of 2".5. The much bigger number of photons detected with the XMM-Newton EPIC and Chandra ACIS S detectors allows us to determine a significantly improved source position.

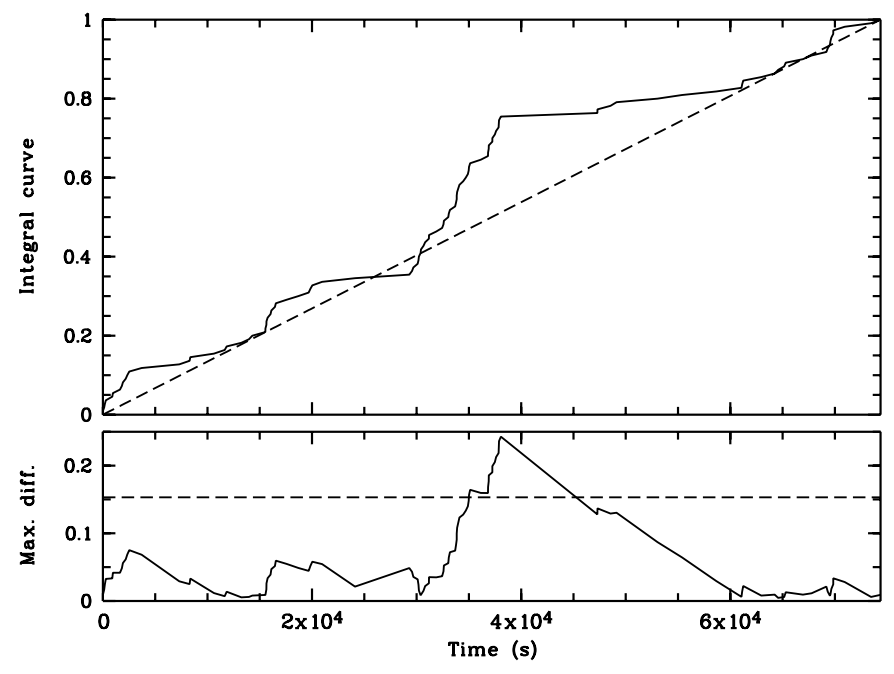

Fig. 4. ROSAT HRI integrated light curve of RX J004717.4-251811 events (selected in a circle around the source with $6^{\prime \prime}$ radius) over the observation time (top). The dashed line shows the expected light curve for a constant source of the same luminosity. The difference between the light curve of the constant source and the observed counts is given below. A 99\% variability threshold according to a KolmogorovSmirnov test is shown as dashed line.

Systematic errors can be reduced by adjusting the positions using the well determined optical ( 0 .' 2 error using USNO-B positions, Monet et al. 2003) and X-ray positions of several QSOs in the field of view.

For Chandra ACIS S observation 790 the VP99 source X22 identified with a QSO (optical blue magnitude of 19.9, redshift 1.25) is detected in chip S3 close to the center of the field of view. During observation 969 it is positioned at a larger off-axis angle in chip S4. For XMM-Newton EPIC besides VP99 X22 the sources X4 and X58 are clearly detected (QSOs with optical blue magnitudes of 20.3 and 18.5 and redshifts of 1.02 and 0.66 , respectively). Averaging the resulting positions from Chandra observations 790 and 969 as well as from the individual EPIC instruments in observation 0110900101 


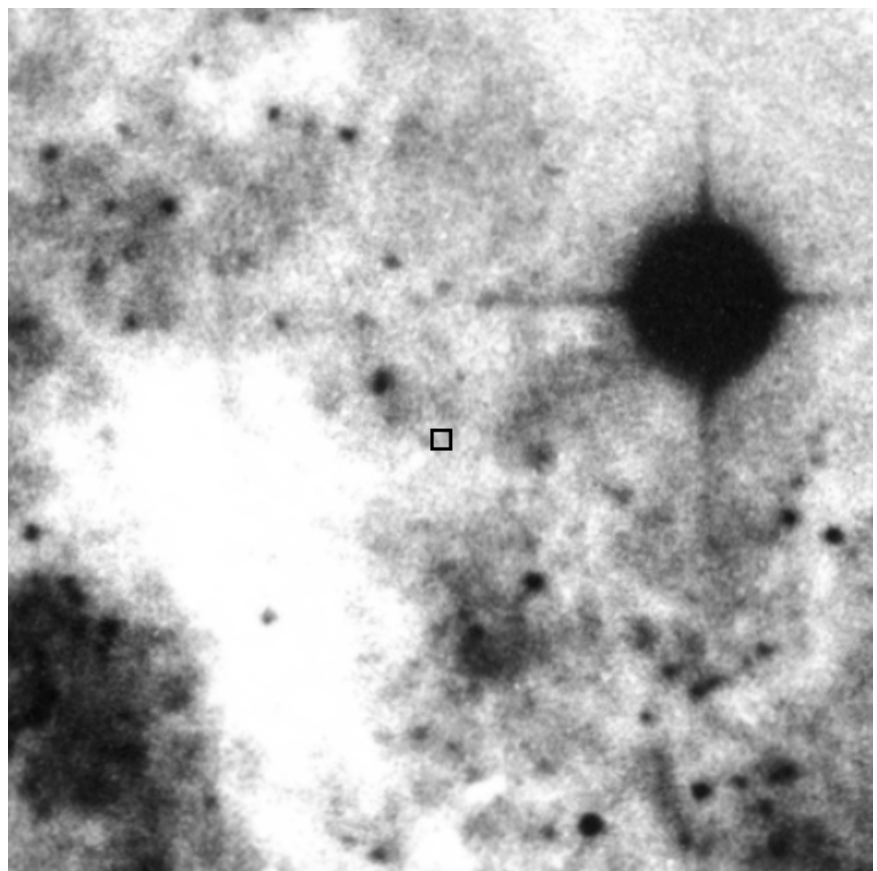

Fig. 5. Position of RX J004717.4-251811 is shown on an extract of a deep blue NGC 253 image (Sofue et al. 1994) covering the same area as Fig. 3 and was kindly provided by Akiko Kawamura. The square ( $1^{\prime \prime}$ at a side) marks the improved position. The image was aligned using USNO-B positions in the field (Monet et al. 2003). The residual error of the solution is about $1^{\prime \prime}$.

we get a highly improved position for RX J004717.4-251811: $\alpha=00^{\mathrm{h}} 47^{\mathrm{m}} 17^{\mathrm{s}} .51, \delta=-25^{\circ} 18^{\prime} 11^{\prime \prime} .2$ (J2000), with an remaining error radius of 0.3 mainly determined by systematics. See Fig. 5 for an overlay of the improved position on a deep optical blue image.

\subsection{Energy spectra}

As discussed in Sect. 2.1, HR changes of RX J004717.4251811 in different brightness regimes indicate spectral variability which may be either caused by reduced absorption during the low intensity states or due to changes of the stiffness of the source spectrum. Unfortunately, the source count rates do not allow a more detailed investigation. For the brighter states of RX J004717.4-251811, that is during Chandra observation 969 as well as during the high state of Chandra observation 790, and XMM-Newton observation 0110900101 more than 600 counts were collected from the source which allowed us a rough spectral analysis. RX J004717.4-251811 photons were extracted using the same areas as for the time variability analysis and spectra were grouped in channels with at least 30 source plus background photons. Due to the limited number of photons we just fitted absorbed one component spectral models: power law (POWL), bremsstrahlung (BREMS), and thin thermal plasma with element abundance fixed to solar (Raymond-Smith, THPL). The resulting integration times, raw count rates and degrees of freedom (DOF) are listed in Table 2 together with the results of the fits.
The absorbed POWL model fits show the lowest $\chi^{2} / v$ values. Absorbing column and photon index $\Gamma$ of all observations coincide within the errors. However, only for the Chandra observations the fit is formally acceptable. Several factors may explain the unacceptably high $\chi^{2} / v$ of the POWL fit for the XMM-Newton EPIC PN spectrum of RX J004717.4-251811. To the one hand, there may be contributions to the spectrum from the nearby source X18. On the other hand, to get sufficient statistics for the fit we accepted all photons in the extraction region, not rejecting events on or close to a CCD column with slightly increased offset which passes through the extraction region. The BREMS spectra exhibit marginally higher $\chi^{2} / v$ values than the POWL fits and find high temperatures (around 7 to $10 \mathrm{keV}$ ). The absorbing column needed in addition to the POWL and BREMS models is $\sim 2 \times 10^{21} \mathrm{~cm}^{-2}$, significantly above the absorption expected within the Milky Way in this direction $\left(1.3 \times 10^{20} \mathrm{~cm}^{-2}\right.$, Dickey \& Lockman 1990). Neither the Chandra nor the XMM-Newton data can be described by a THPL spectrum.

The best fit POWL model parameters of Chandra observation 969 are used in Table 1 for conversion from count rates to intrinsic source luminosities for observations of all observatories. We are aware that the smaller HR values during low intensity states indicate a higher low energy flux and therefore a different spectrum. This may lead to an overestimation of the intrinsic low state luminosities in the table.

\section{Orbital period determination}

The intensity changes of RX J004717.4-251811 from low to high in the XMM-Newton observation 0110900101 and in the Chandra observation 790 are not resolved by the $1000 \mathrm{~s}$ time resolution of our plots. Shorter time bins do not help as the source is not bright enough. In the following we interpret the intensity changes as egresses from eclipse of an XRB. The times of egresses have to be separated by an integer number of periods and therefore give a first constrain on the possible orbital period $p$ of RX J004717.4-251811:

$p=(352.870 \pm 0.012) \mathrm{d} / n ; n=1,2,3, \ldots$

without exactly knowing the number $n$ of periods between the two egresses. The relative period error is $3.3 \times 10^{-5}$.

The Chandra observation 790 is the longest continuous observation of RX J004717.4-251811 covering 0.5092 d, however only part of an orbit. This together with Eq. (1) constrains the number of possible periods to 692 (covering the range $0.5099 \mathrm{~d}$ and $352.870 \mathrm{~d})$.

During Chandra observation 969 the flux of RX J004717.4251811 was constant within the errors and even brighter than during the 790 bright state. The source therefore undoubtedly was out of eclipse. We use the conditions that the noneclipse observations are not allowed to include orbit phases covering the minimum eclipse duration $(0.14 \mathrm{~d}$ during the Chandra observation) and assuming that the eclipse phase is longer than 0.13 . This constrains the number of possible periods to 457 (between $0.5129 \mathrm{~d}$ and $70.574 \mathrm{~d}$ ).

We can try to further reduce the number of acceptable periods with the help of the other XMM-Newton and 
Table 2. Spectral modeling results for RX J004717.4-251811. Only Chandra ACIS S and XMM-Newton EPIC PN times in high state were used. In the case of $\chi^{2} / v \leq 2.0,90 \%$ errors are given.

\begin{tabular}{lrrrlrrrrr}
\hline \hline Observation & $\begin{array}{r}T_{\text {int }} \\
(\mathrm{ks})\end{array}$ & $\begin{array}{r}\text { Raw count rate } \\
\left(10^{-2} \mathrm{ct} \mathrm{s}^{-1}\right)\end{array}$ & DOF & Model & $\begin{array}{r}N_{\mathrm{H}} \\
\left(10^{21} \mathrm{~cm}^{-2}\right)\end{array}$ & $\Gamma$ & $\begin{array}{r}T \\
(\mathrm{keV})\end{array}$ & $\begin{array}{r}L_{\mathrm{X}}^{* *} \\
\left(10^{38} \mathrm{erg} \mathrm{s}^{-1}\right)\end{array}$ & $\chi^{2} / v$ \\
\hline XMM-Newton & 16.5 & $3.8 \pm 0.7$ & 17 & POWL & $2.3_{-0.7}^{+1.0}$ & $1.9_{-0.3}^{+0.2}$ & & $2.6_{-0.6}^{+0.7}$ & 1.63 \\
EPIC PN & & & & BREMS & $1.6_{-0.6}^{+0.8}$ & & $6_{-2}^{+4}$ & $2.3_{-0.4}^{+0.4}$ & 1.77 \\
0110900101 & & & & THPL & 12 & & 1.0 & 5.8 & 7.1 \\
Chandra & 14.2 & $5.7 \pm 0.3$ & 21 & POWL & $1.9_{-0.8}^{+0.8}$ & $1.7_{-0.2}^{+0.2}$ & & $4.1_{-0.7}^{+1.1}$ & 0.69 \\
969 & & & & BREMS & $1.2_{-0.6}^{+0.7}$ & & $8_{-3}^{+6}$ & $3.9_{-0.4}^{+0.4}$ & 0.69 \\
& & & & THPL & 10 & & 1.0 & 6.9 & 9.7 \\
Chandra & 31.0 & \multirow{2}{*}{$3.2 \pm 0.1$} & 26 & POWL & $1.8_{-0.9}^{+1.0}$ & $1.8_{-0.2}^{+0.2}$ & & $3.0_{-0.7}^{+0.7}$ & 1.10 \\
790 & & & & BREMS & $1.0_{-0.7}^{+0.7}$ & & $8_{-3}^{+5}$ & $2.7_{-0.3}^{+0.3}$ & 1.11 \\
& & & & THPL & 9.0 & & 1.0 & 4.5 & 6.3 \\
\hline
\end{tabular}

${ }^{*}$ THPL $=$ thin thermal Plasma with solar abundance, BREMS = thermal bremsstrahlung, POWL = power law.

${ }^{* *}$ In the $0.5-7.5 \mathrm{keV}$ band, corrected for total absorption, corrected for extraction radii and vignetting.

Chandra observations and in addition use the sparse ROSAT and Einstein HRI photons. This selection however has to be taken with care as it is hampered by the limited knowledge of the luminosity state of RX J004717.4-251811 during these observations and the limited photon statistics.

XMM-Newton observations 0125960101 (duration $0.451 \mathrm{~d})$ and $0125960201(0.164 \mathrm{~d})$ were separated by $0.174 \mathrm{~d}$. RX J004717.4-251811 was again constant within the errors during each observation and also from observation to observation, however only a factor of 1.9 brighter than during eclipse of 0110900101 with comparable hardness ratio values. As the residual flux during eclipse may vary from eclipse to eclipse similar to the source flux out of eclipse we cannot decide if the observations are out of eclipse (I) or if one eclipse covers both observations (II). The condition (I) reduces the number of allowed periods to 141 (between $0.6416 \mathrm{~d}$ and $70.574 \mathrm{~d}$ ). If we assume for condition (II) a maximum eclipse duration of 0.25 in phase (similar to that reported for Cen X-3), the period has to be longer than $3.156 \mathrm{~d}$, the two observations at the right phase and Chandra 969 not within the same phase interval. The condition (II) reduces the number of allowed periods to 10 (between $4.1031 \mathrm{~d}$ and $32.079 \mathrm{~d}$ ).

Also for Chandra observations 383 we cannot decide if the source was in or out of eclipse as the source is only a factor of two brighter than during eclipse of 790 and shows similar hardness ratio. For 23 of the 141 period candidates of condition (I) and 3 of the 10 for (II) the source would be in eclipse during Chandra observation 383.

The detection of two eclipse egresses within the few XMMNewton and Chandra observations of less than $0.5 \mathrm{~d}$ duration strongly favors a short orbital period of RX J004717.4-251811. If we constrain the period to less than $10 \mathrm{~d}$ there only remain 118 periods for condition (I) and 5 for (II) to be investigated. During 18 (1) of those RX J004717.4-251811 would be in eclipse during Chandra observation 383.

To further reduce the number of acceptable periods we folded the ROSAT HRI counts detected from the
RX J004717.4-251811 area over the period ranges allowed by the candidate periods. We checked if the source could have remained in low intensity (eclipse) for at least the duration of the measured longest low states $(0.14 \mathrm{~d})$ or for 0.13 of the candidate period and if this low intensity time was compatible in phase with the Chandra eclipse egress for period candidates derived with condition (I). For condition (II) the eclipse had to last as long as indicated by the XMM-Newton observations. Many of the candidate periods could be rejected using these criteria. Some periods cannot be rejected because the expected eclipse phase is not covered by ROSAT exposure. Some periods however show only background counts at the expected eclipse phases even when the ROSAT exposure is high. All periods selected under condition (II) are not acceptable using these criteria. Under condition (I), we get 14 candidate periods with a lower error range. During three of the periods, RX J004717.4251811 is in eclipse during Chandra observation 383.

In a last step, the candidate periods were checked for consistency with the Einstein observations (collected during a RX J004717.4-251811 high state in July 1979, see Table 1). Only seven periods, two with Chandra observation 383 in eclipse, pass this test (see Table 3 ). The periods above 5 days are less probable as the chance to detect two eclipse egresses during the XMM-Newton and Chandra observations would have been low. From the remaining periods the ones with allowed eclipse duration longer than 0.13 in phase seem to be more probable.

Taking all the selection criteria into account $1.470243 \mathrm{~d}$ seems to be the best period candidate followed by $3.207928 \mathrm{~d}$. Additional observations of RX J004717.4-251811 in the high state are urgently needed to finally decide on the correct orbital period.

\section{Discussion}

In the last sections we have shown that the variety of observations of RX J004717.4-251811 can be explained in an 
Table 3. Allowed orbital periods of RX J004717.4-251811.

\begin{tabular}{lrrrr}
\hline \hline Period & $\begin{array}{r}\text { Error* } \\
\left(10^{-6} \mathrm{~d}\right)\end{array}$ & $\begin{array}{r}\text { Ecl. dur. } \\
\left({ }^{* *}\right)\end{array}$ & $\begin{array}{r}\text { Obs. 383 } \\
\left({ }^{* *}\right)\end{array}$ & $\begin{array}{r}\text { Comment } \\
\left({ }^{* * * *}\right)\end{array}$ \\
\hline 1.470243 & 10 & 0.15 & & $\mathrm{~B}$ \\
2.484902 & 10 & 0.13 & & $\mathrm{~B}$ \\
2.778391 & 10 & 0.13 & $\mathrm{E}$ & $\mathrm{B}$ \\
3.207928 & 10 & 0.14 & & \\
4.969849 & 20 & 0.13 & $\mathrm{E}$ & $\mathrm{B}$ \\
6.190937 & 20 & 0.14 & & B, E- \\
7.671308 & 20 & 0.15 & & E- \\
\hline
\end{tabular}

Notes:

* Determined to achieve longest possible eclipse duration.

** Maximum allowed eclipse duration ( $\Delta$ phase).

*** E if RX J004717.4-251811 in eclipse during Chandra 383.

${ }^{* * * *}$ B period at boundary of allowed window, E- no Einstein exposure during eclipse.

eclipsing XRB scenario. RX J004717.4-251811 is the first eclipsing XRB identified outside the Local Group.

The length of eclipse and the luminosity during its high state of $\sim 2 \times 10^{38} \mathrm{erg} \mathrm{s}^{-1}$, i.e. at the Eddington limit of a $1.4 M_{\odot}$ neutron star, are most easily explained by a HMXB with a neutron star as companion. Similar luminosities were reported for the eclipsing HMXBs SMC X-1 and LMC X-4 during the high state. Detection of pulsations in the tens of second range or shorter are expected in such systems. For a faint source like RX J004717.4-251811 such short pulsations are difficult to detect as time delays of photon arrival times due to the motion in the binary orbit will smear out the periodic signal.

Accreting XRBs normally show pulsation averaged X-ray energy spectra above $1 \mathrm{keV}$ that can be modeled by power laws with high energy cut-offs. The photon indices of the power law spectra range between 0.8 and 1.5 for most pulsars, but some sources show rather soft spectra (see the review of Nagase 1989). The steep power law slope of 1.9 in the brighter states puts RX J004717.4-251811 in this special category.

For most eclipsing XRB residual emission during eclipse was measured. The emission can be explained by re-processing of primary X-rays originating from the bright area at the magnetic poles of the neutron star, in an extended accretion disk corona (which is not fully occulted) or by scattering in the companion atmosphere/stellar wind. Residual emission of up to $\sim 10 \%$ was reported (Ebisawa et al. 1996; Haberl 1991) depending on system geometry and wind density. The RX J004717.4-251811 residual emission of $~ 5 \%$ is well within these limits.

As discussed in Sect. 3, RX J004717.4-251811 was most likely out of eclipse during the XMM-Newton observations in December 2000 and the Chandra observation in August 2001. During these observations the source luminosity was up to a factor of ten lower than during the high states (luminosities close to Eddington limit) indicating clear long term time variability. During the Einstein observations in July 1979 and also during the ROSAT observations in January 1995 the source was in high state, while during the rest of the ROSAT observations the intensity was significantly lower (see Fig. 4). Similar long term variability was detected in several other low and high mass XRBs where it even may manifest itself in a long term superorbital period (e.g. Her X-1, LMC X-4, SMC X1) typically on the scale of tens of days. Due to the up to now sparse time coverage of RX J004717.4-251811 observations, such long term periodicities were not detected, but at present also cannot be ruled out. The Her X-1 and LMC X4 long term periodicities correspond to $\sim 21$ orbital periods. If a RX J004717.4-251811 long term variability follows a similar pattern, the most probable orbital period of $1.47 \mathrm{~d}$ would lead to $\sim 31 \mathrm{~d}$ as period of the long term variability.

During some ROSAT observation intervals of typically $1000 \mathrm{~s}$ duration there are indications of short term flaring (see Fig. 4). Similar flares up to now were not detected during XMM-Newton and Chandra observations. Short term flaring was detected in several HMXBs (e.g. LMC X-4, Pietsch et al. 1985).

The most promising orbital periods of RX J004717.4251811 do not allow us to decide if the optical companion is a high or low mass star. LMC X-4 and SMC X-1 are HMXB systems with orbital periods of $1.4 \mathrm{~d}$ and $3.9 \mathrm{~d}$, Her X-1 with an intermediate period $(1.7 \mathrm{~d})$ on the other hand is a LMXB. The X-ray luminosity of RX J004717.4-251811 in the bright state at or above the Eddington limit and possible flaring (see above) may indicate a HMXB as the LMXB Her X-1 only reaches about one third of the Eddington luminosity (Forman et al. 1972; Howarth \& Wilson 1983) and does not show flares.

If we therefore assume that RX J004717.4-251811 is a HMXB we can use the optical magnitudes of the known systems in the Magellanic Clouds to determine the optical brightness in NGC 253. The optical brightnesses of LMC X-4 and SMC X-1 - V of $14.0 \mathrm{mag}$ and $13.3 \mathrm{mag}$ (Liu et al. 2000) and distances of $50 \mathrm{kpc}$ and $59 \mathrm{kpc}$, respectively (see review by van den Bergh 1999) - correspond to (21.4-22.6) mag at the assumed distance of $2.58 \mathrm{Mpc}$ to NGC 253. If this distance has to be corrected to $3.94 \mathrm{Mpc}$ as recently indicated by Karachentsev et al. (2003), not only the estimated optical magnitudes would have to be increased by 0.9 mag but also the derived X-ray luminosities for RX J004717.4-251811 would rise by a factor of 2.3. The expected optical magnitude may have to be increased by up to one optical magnitude if the absorption measured in the X-ray spectrum in the high state $\left(N_{\mathrm{H}}=1.9 \times 10^{21} \mathrm{~cm}^{-2}\right.$, see above $)$ is due to extinction within NGC 253 and not due to absorption in the near surrounding of the X-ray emitting area within the binary system (Predehl \& Schmitt 1995). Additional extinction within NGC 253 may well be present as RX J004717.4-251811 seems to be positioned at the edge of a dark cloud (see Fig. 5). A preliminary analysis of the image indicates that stars of visual magnitude 23 should just be visible. There is a faint object at the east edge of the error box which could be the optical counterpart. However, if RX J004717.4-251811 is a LMXB similar to Her X-1 (14 mag at a distance of $6 \mathrm{kpc}$, see e.g. Howarth \& Wilson 1983), it would be fainter than $27 \mathrm{mag}$ at the distance of NGC 253, very difficult to be separated from the many other 
stars of similar brightness in the NGC 253 surrounding, and not be detectable in current optical imaging. Only deeper time resolved imaging and spectroscopy can lead to a secure identification of RX J004717.4-251811as a HMXB and solve the high mass - low mass ambiguity.

\section{Conclusions and outlook}

Starting from an XMM-Newton observation in which RX J004717.4-251811 changed state from low to high, we found a similar transition in a Chandra observation. Interpreted as eclipse egresses we determined candidate orbital periods for the source. With the help of archival XMM-Newton, Chandra, ROSAT and Einstein observations we selected as most probable orbital periods $1.47029 \mathrm{~d}$ and $3.20793 \mathrm{~d}$. RX J004717.4251811 is one of the few eclipsing X-ray binary systems and the first detected outside the Local Group. No pulsation period was found. The source is clearly variable on time scales of tens of days and also short time flaring (1000 s time scale) is indicated. The energy spectrum of the source during the high state can be described by a hard power law spectrum with absorption significantly higher than the foreground absorption in the Milky Way. Using the improved source position we indicate a possible optical identification. RX J004717.4-251811 is most likely a HMXB system similar to SMC X-1 and LMC X-4.

Two long observations of NGC 253 accepted for the XMMNewton and Chandra program (140 ks and $85 \mathrm{ks}$, respectively) hopefully will detect RX J004717.4-251811 in the high state and allow us to finally decide on the orbital period of the source and enable sensitive neutron star rotation searches from seconds to tens of seconds. The XMM-Newton observation would cover one full binary orbit if the $1.5 \mathrm{~d}$ period is correct and finally clarify the correct orbital solution. A search for sub-second periods will only be possible in dedicated observations of RX J004717.4-251811 with higher time resolution (that could not fully cover the galaxy).

Acknowledgements. The XMM-Newton project is supported by the Bundesministerium für Bildung und Forschung/Deutsches Zentrum für Luft- und Raumfahrt (BMBF/DLR), the Max-Planck Society and the Heidenhain-Stiftung.

\section{References}

Buccheri, R., Bennett, K., Bignami, G. F., et al. 1983, A\&A, 128, 245

Dickey, J. M., \& Lockman, F. J. 1990, ARA\&A, 28, 215

Ebisawa, K., Day, C. S. R., Kallman, T. R., et al. 1996, PASJ, 48, 425

Fabbiano, G., \& Trinchieri, G. 1984, ApJ, 286, 491 (FT84)

Forman, W., Jones, C. A., \& Liller, W. 1972, ApJ, 177, L103

Haberl, F. 1991, A\&A, 252, 272

Haberl, F., \& Zavlin, V. E. 2002, A\&A, 391, 571

Howarth, I. D., \& Wilson, B. 1983, MNRAS, 202, 347

Jansen, F., Lumb, D., Altieri, B., et al. 2001, A\&A, 365, L1

Karachentsev, I. D., Grebel, E. K., Sharina, M. E., et al. 2003, A\&A, accepted [astro-ph0302045]

Li, F., Rappaport, S., \& Epstein, A. 1978, Nature, 271, 37

Liu, Q. Z., van Paradijs, J., \& van den Heuvel, E. P. J. 2000, A\&AS, 147,25

Monet, D. G., Levine, S. E., Canzian, B., et al. 2003, AJ, 125, 984

Nagase, F. 1989, PASJ, 41, 1

Peres, G., Reale, F., Collura, A., \& Fabbiano, G. 1989, ApJ, 336, 140

Pietsch, W., Pakull, M., Voges, W., \& Staubert, R. 1985, Space Sci. Rev., 40, 23

Pietsch, W., Roberts, T. P., Sako, M., et al. 2001, A\&A, 365, L174 (P01)

Predehl, P., \& Schmitt, J. H. M. M. 1995, A\&A, 293, 889

Puche, D., Carignan, C., \& van Gorkom, J. H. 1991, AJ, 101, 456

Schreier, E., Giacconi, R., Gursky, H., Kellogg, E., \& Tananbaum, H. 1972a, ApJ, 178, L71

Schreier, E., Levinson, R., Gursky, H., et al. 1972b, ApJ, 172, L79

Sofue, Y., Wakamatsu, K., \& Malin, D. F. 1994, AJ, 108, 2102

Strüder, L., Briel, U., Dennerl, K., et al. 2001, A\&A, 365, L18

Tananbaum, H., Gursky, H., Kellogg, E. M., et al. 1972, ApJ, 174, L143

Turner, M. J. L., Abbey, A., Arnaud, M., et al. 2001, A\&A, 365, L27

van den Bergh, S. 1999, in New Views of the Magellanic Clouds, IAU Symp., 190, 569

van Paradijs, J., \& McClintock, J. E. 1995, in X-ray Binaries, ed. W. H. G. Lewin, J. van Paradijs, \& E. P. J. van den Heuvel (Cambridge: Cambridge Univ. Press), 58

Vogler, A., \& Pietsch, W. 1999, A\&A, 342, 101 (VP99)

White, N. E. 1978, Nature, 271, 38 